\title{
Efficient Nonfullerene Organic Solar Cells with Small Driving Forces for Both Hole and Electron Transfer
}

Shangshang Chen, Yuming Wang, Lin Zhang, Jingbo Zhao, Yuzhong Chen, Danlei Zhu, Huatong Yao, Guangye Zhang, Wei Ma, Richard H. Friend, Philip C. Y. Chow, Feng Gao and He Yan

The self-archived postprint version of this journal article is available at Linköping University Institutional Repository (DiVA):

http:/ / urn.kb.se/ resolve?urn=urn:nbn:se:liu:diva-153181

N.B.: When citing this work, cite the original publication.

Chen, S., Wang, Y., Zhang, L., Zhao, J ., Chen, Y., Zhu, D., Yao, H., Zhang, G., Ma, W., Friend, R. H., Chow, P. C. Y., Gao, F., Yan, He, (2018), Efficient Nonfullerene Organic Solar Cells with Small Driving Forces for Both Hole and Electron Transfer, Advanced Materials, 30(45), 1804215.

https:// doi.org/ 10.1002/ adma.201804215

Original publication available at:

https:// doi.org/ 10.1002/ adma.201804215

Copyright: Wiley (12 months)

http:/ / eu.wiley.com/WileyCDA/ 


\section{Efficient Non-Fullerene Organic Solar Cells with Small Driving Forces for Both Hole and Electron Transfers}

Shangshang Chen, ${ }^{\S}$ Yuming Wang, ${ }^{\S}$ Lin Zhang, Jingbo Zhao, Yuzhong Chen, Danlei Zhu, Huatong Yao, Guangye Zhang, Wei Ma, Richard H. Friend, Philip C. Y. Chow, ${ }^{*}$ Feng Gao,* and He Yan*

$\S$ S. Chen, Y. Wang

These authors contributed equally to this work.

S. Chen, Dr. J. Zhao, Y. Chen, H. Yao, Dr. G. Zhang, Dr. P. C. Y. Chow, Prof. H. Yan Department of Chemistry, Energy Institute and Hong Kong Branch of Chinese National Engineering Research Center for Tissue Restoration \& Reconstruction, Hong Kong University of Science and Technology (HKUST), Clear Water Bay, Kowloon, Hong Kong. E-mail: hyan@ust.hk; pcyc@ust.hk

Y. Wang, Prof. F. Gao

Department of Physics, Chemistry and Biology (IFM), Linköping University, Linköping SE58183, Sweden.

Email: fenga@ifm.liu.se

L. Zhang, Prof. W. Ma

State Key Laboratory for Mechanical Behavior of Materials, Xi'an Jiaotong University, Xi'an 710049, P. R. China.

D. Zhu

Institute of Chemistry, Chinese Academy of Sciences, Beijing, 100190, P. R. China.

Prof. R. H. Friend

Cavendish Laboratory, J J Thomson Avenue, Cambridge CB3 0HE, UK.

Dr. P. C. Y. Chow, Prof. H. Yan

HKUST-Shenzhen Research Institute, No. 9 Yuexing 1st RD, Hi-tech Park, Nanshan, Shenzhen 518057, China.

Keywords: organic solar cells, small-molecular acceptors, voltage loss, charge transfer

State-of-the-art organic solar cells (OSCs) typically suffer from large voltage loss ( $\left.V_{\text {loss }}\right)$ compared to their inorganic and perovskite counterparts. There are some successful attempts to reduce the $V_{\text {loss }}$ by decreasing the energy offsets between donor and acceptor materials, and the OSC community has demonstrated efficient systems with either small HOMO offset or negligible LUMO offset between donors and acceptors. However, the efficient OSCs based on a donor/acceptor system with both small HOMO and LUMO offsets have not been 
demonstrated simultaneously. In this work, we report an efficient non-fullerene OSC based on a donor polymer named PffBT2T-TT and a small-molecular acceptor (O-IDTBR) with identical bandgaps and close energy levels. Fourier-transform photocurrent spectroscopy external quantum efficiency spectrum of the blend overlaps with those of neat PffBT2T-TT and O-IDTBR, indicating small driving forces for both hole and electron transfers. Meanwhile, the OSCs exhibited a high electroluminescence quantum efficiency (EQE $E_{\mathrm{EL}}$ ) of $\sim 1 \times 10^{-4}$, which leads to a significantly minimized non-radiative $V_{\text {loss }}$ of $0.24 \mathrm{~V}$. Despite the small driving forces and a low $V_{\text {loss, }}$ a maximum EQE of $67 \%$ and a high power conversion efficiency of $10.4 \%$ can still be achieved.

Bulk-heterojunction (BHJ) organic solar cells (OSCs) based on electron donors and electron acceptors have attracted extensive research interests from both academia and industry due to their advantages of light weight, mechanical flexibility, and compatibility with roll-to-roll printing processes. ${ }^{[1-4]}$ Although fullerene derivatives have been the dominant acceptor materials for nearly two decades, non-fullerene OSCs have emerged as the new generation solar cell technology. ${ }^{[5-16]}$ One of the main advantages of non-fullerene OSCs is the reduced voltage loss ( $V_{\text {loss }}$ ), which is defined as the difference between the optical bandgap of donor/acceptor $\left(E_{\text {gap,D/A }}\right)$ and the device open-circuit voltage $\left(V_{\text {oc }}\right)$, and is a key parameter that determines the maximum achievable power conversion efficiency (PCE) for any type of solar cells. ${ }^{[12,17-19]}$ Currently, the best performing fullerene OSC with a certified PCE of $11.5 \%$ has a large $V_{\text {loss }}$ of $0.87 \mathrm{~V},{ }^{[4]}$ which is much greater than those typically found in inorganic and perovskite cells $(\sim 0.5 \mathrm{~V}) .{ }^{[20]}$ Previous studies have reported several cases of fullerene OSCs with reduced $V_{\text {loss. }}{ }^{[21-22]}$ However, these systems typically suffer from poor charge generation efficiency and/or low fill-factor $(F F)$ that outweigh the benefit of the improved $V_{\text {oc. }}$. Alternatively, state-of-the-art non-fullerene OSCs can achieve low $V_{\text {loss }}$ while exhibiting 
efficient charge generation. For example, a BHJ blend of P3TEA:SF-PDI 2 can achieve a small $V_{\text {loss }}$ of $0.61 \mathrm{~V}$ and still maintain efficient charge generation (a maximal EQE of $66 \%) .{ }^{[17]}$ Therefore, the development of non-fullerene OSCs with small $V_{\text {loss }}$ offers a promising pathway for further improving OSC performance.

The reduction in $V_{\text {loss }}$ is typically achieved by decreasing the energy offset in either the lowest unoccupied molecular orbital (LUMO) or the highest occupied molecular orbital (HOMO) levels between the donor and the acceptor. In the case based on P3TEA:SF-PDI 2 , the small LUMO offset, more precisely, the offset between the bandgap of the donor (P3TEA) and the energy of the charge transfer (CT) states of the blend ( $\left.E_{\text {gap }, ~}-E_{\mathrm{CT}}\right)$, is commonly regarded the driving force of the electron transfer from the donor to the acceptor. ${ }^{[23]}$ Importantly, both hole and electron transfers are important processes that can make significant contribution to photocurrent generation in non-fullerene OSCs, because non-fullerene acceptors are highly absorptive compared to fullerene derivatives. ${ }^{[24]}$ The excitons generated by non-fullerene acceptors can also be separated at donor/acceptor interfaces via the hole transfer from the acceptor to the donor, thus contributing to the overall charge generation. ${ }^{[25]}$ In this case, the HOMO offset, more precisely, the offset between the bandgap of the acceptor and the energy

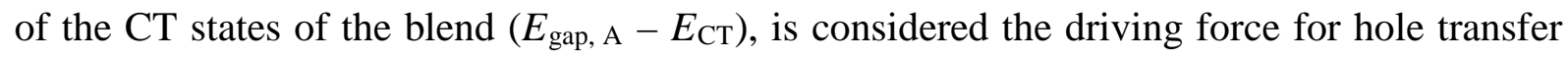
from the acceptor to the donor.

Thus far, there are two types of non-fullerene OSCs with small driving forces that have been reported. One type has small LUMO but large HOMO offsets (e.g. P3TEA:SF-PDI 2 ), while the other type has small HOMO but large LUMO offsets. ${ }^{[18,26-28]}$ The OSC community has demonstrated that efficient charge separation can be achieved as long as one of the two energy offsets (HOMO or LUMO) is small. However, a system that can exhibit efficient charge generation with small LUMO and HOMO energy offsets have not been demonstrated. To prove that the charge separation in OSCs truly does not require large driving force for both 
holes and electrons, it would be important to demonstrate a donor:acceptor system, in which the driving forces for both electron and hole transfers are small.

Here we report, for the first time, an efficient non-fullerene OSC based on a new donor polymer (named PffBT2T-TT) and a small-molecular acceptor (SMA) named O-IDTBR ${ }^{[29]}$ with both small driving forces for hole and electron transfers (Scheme 1). The PffBT2T-TT polymer has an identical optical bandgap and close energy levels to those of O-IDTBR. The Fourier-transform photocurrent spectroscopy external quantum efficiency (FTPS-EQE) spectrum of the blend overlaps with those of neat PffBT2T-TT and O-IDTBR, indicative of both negligible $E_{\text {gap, D }}-E_{\mathrm{CT}}$ and $E_{\text {gap }}$ A $-E_{\mathrm{CT}}$ offsets. Meanwhile, the PffBT2T-TT:OIDTBR-based OSCs exhibited a high electroluminescence quantum efficiency (EQE $\left.E_{\mathrm{EL}}\right)$ of $\sim 1 \times 10^{-4}$, leading to a significantly minimized non-radiative $V_{\text {loss }}$ of $0.24 \mathrm{~V}$. Despite the negligible driving forces and a small $V_{\text {loss, }}$ a maximum EQE of $67 \%$ can still be achieved in the OSCs based on PffBT2T-TT:O-IDTBR. Combined with a favorable morphology featured with high crystallinity and small domain size, the PffBT2T-TT:O-IDTBR-based OSCs realize a high PCE up to $10.4 \%$, and a high $V_{\text {oc }}$ of $1.08 \mathrm{~V}$, corresponding to an overall $V_{\text {loss }}$ as small as $0.55 \mathrm{~V}$. Fundamentally, our system is different from the previously reported low- $V_{\text {loss }}$ OSCs achieved from either small $E_{\text {gap, D }}-E_{\text {CT }}$ or $E_{\text {gap, A }}-E_{\text {CT }}$ offset, and our work demonstrates that efficient charge transfer can occur despite both negligible $E_{\text {gap, D }}-E_{\text {CT }}$ and $E_{\text {gap , A }}-E_{\text {Cт }}$ offsets, which will have significant impacts on understanding the charge transfer process of OSCs.

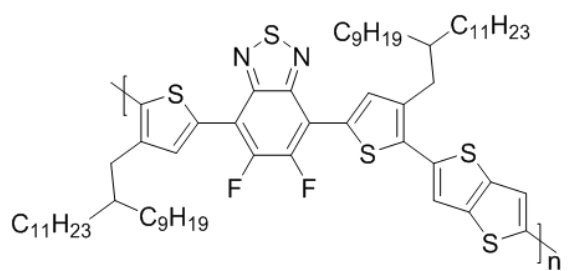

PffBT2T-TT

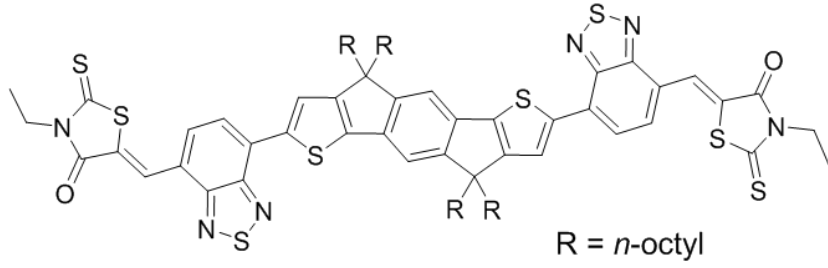

O-IDTBR

Scheme 1. Chemical structures of PffBT2T-TT and O-IDTBR. 
(a)

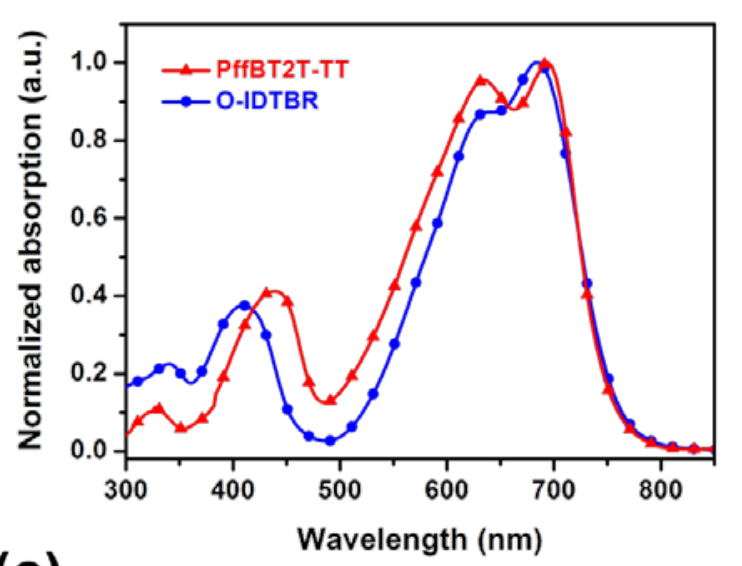

(c)

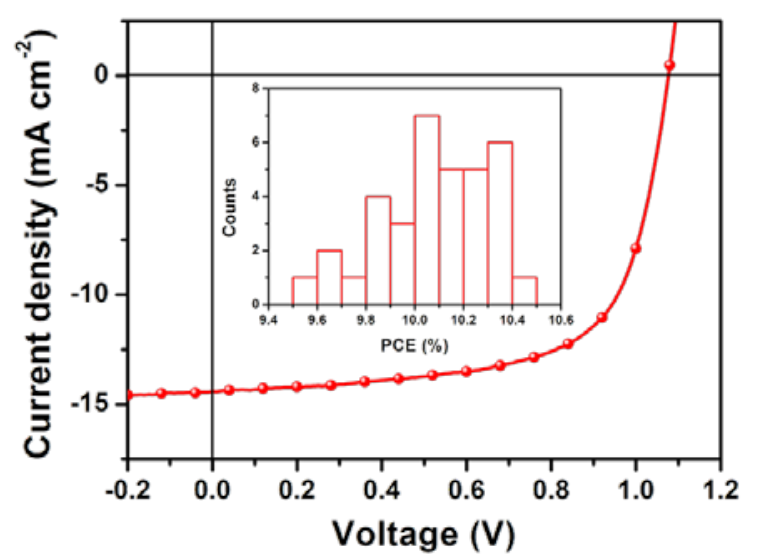

(b)

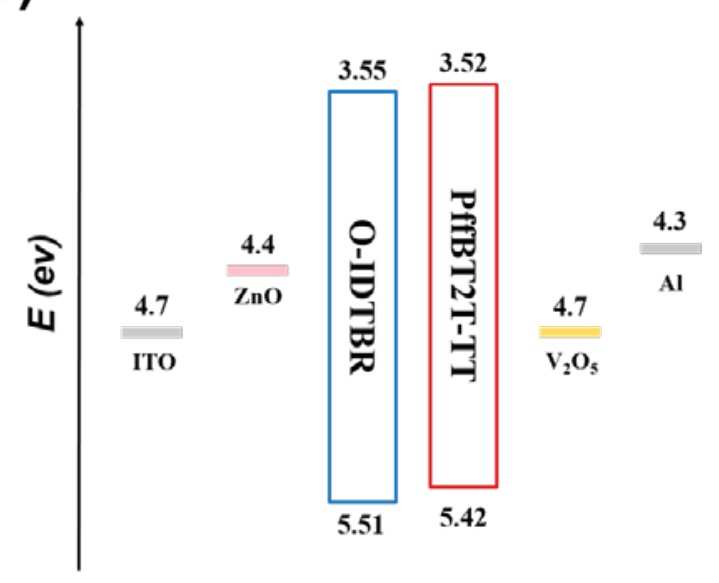

(d)

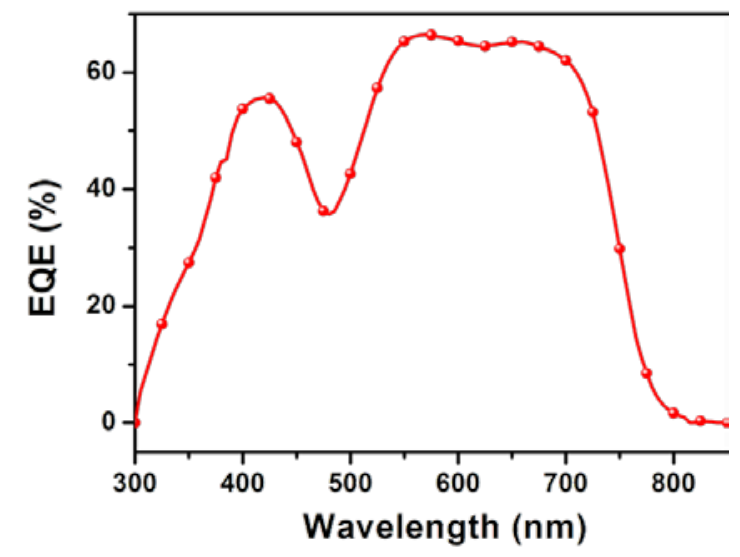

Figure 1. a) Normalized UV-Vis absorption spectra of the neat films. b) Energy diagram representing each layer used in the inverted device architecture. c) $J$ - $V$ characteristics under AM 1.5G illumination (100 $\left.\mathrm{mW} \mathrm{cm}^{-2}\right)$; the inset shows the histogram of the PCE counts for 35 devices. d) EQE spectrum of the optimized PffBT2T-TT:O-IDTBR device.

The detailed description of synthesis of PffBT2T-TT can be found in the Supporting Information, and PffBT2T-TT exhibits a nearly identical optical bandgap to O-IDTBR (1.63 $\mathrm{eV}$, estimated from the onset of the film absorptions) as shown in Figure 1a. The energy levels of both donor and acceptor components were investigated by cyclic voltammetry $(\mathrm{CV}$, Figure S1a) method, and both HOMO and LUMO offsets between PffBT2T-TT and OIDTBR are quite small $(<0.10 \mathrm{eV}$, Figure $1 \mathrm{~b})$. The photovoltaic performance based on the 
PffBT2T-TT:O-IDTBR blend was tested with an inverted device architecture of glass/ITO/ZnO/PffBT2T-TT:O-IDTBR/ $\mathrm{V}_{2} \mathrm{O}_{5} / \mathrm{Al}$, and a high PCE up to $10.4 \%$ was achieved with a high $V_{\text {oc }}$ of $1.08 \mathrm{~V}$, a short-circuit current density $\left(J_{\mathrm{sc}}\right)$ of $14.32 \mathrm{~mA} \mathrm{~cm}{ }^{-2}$, and a high fill factor (FF) of 0.67 (Figure 1c and Table S1). The $V_{\text {loss }}$ in this system was estimated to be $0.55 \mathrm{~V}$ calculated from the absorption onset of the blend film and the $V_{\text {oc }}$ of the OSCs, which is one of the lowest $V_{\text {loss }}$ among the OSCs with PCEs over $10 \%$. Despite such a small $V_{\text {loss, }}$, the system can still achieve efficient charge separation evidenced by the EQE spectrum shown in Figure 1d. The optimized device yielded a broad photon-to-current response from 300 to $800 \mathrm{~nm}$, with EQE values exceeding $60 \%$ from 520 to $720 \mathrm{~nm}$, and a peak value of $67 \%$ at 570 nm (contributed by both PffBT2T-TT and O-IDTBR).

The high EQE values indicate that the efficient charge transfer can occur despite the close energy levels of donor and acceptor components. As reported in the recent work, the energy offset should be characterized by $E_{\text {gap,D/A }}-E_{\mathrm{CT}}$ as measured by the blend, instead of the offset between HOMO or LUMO energy levels measured separated for donor and acceptor materials. ${ }^{[30-31]}$ Therefore, we use $E_{\text {gap, D }}-E_{\text {CT }}$ and $E_{\text {gap, A }}-E_{\text {CT }}$ as energy offsets for electron and hole transfers hereinafter. It is important to note that, despite the small energy difference between the donor and acceptor materials in this blend, the D/A interface remains critical for facilitating charge generation. This is reflected by the poor efficiency of devices with active layers comprising only one of the two materials (Table S2). It is also worthwhile mentioning that the PCE of PffBT2T-TT:O-IDTBR based OSCs is mainly limited by the device $J_{\mathrm{sc}}$, while the $V_{\text {oc }}$ and $F F$ are relatively high among the reported efficient non-fullerene OSCs. We note that the device $J_{\mathrm{sc}}$ is limited by the overlapping absorptions of PffBT2T-TT and O-IDTBR, which is not ideal for solar cell application due to the limited absorption range. Nevertheless, this material system provides an ideal platform to study the charge transfer and other photophysics processes in OSCs. 

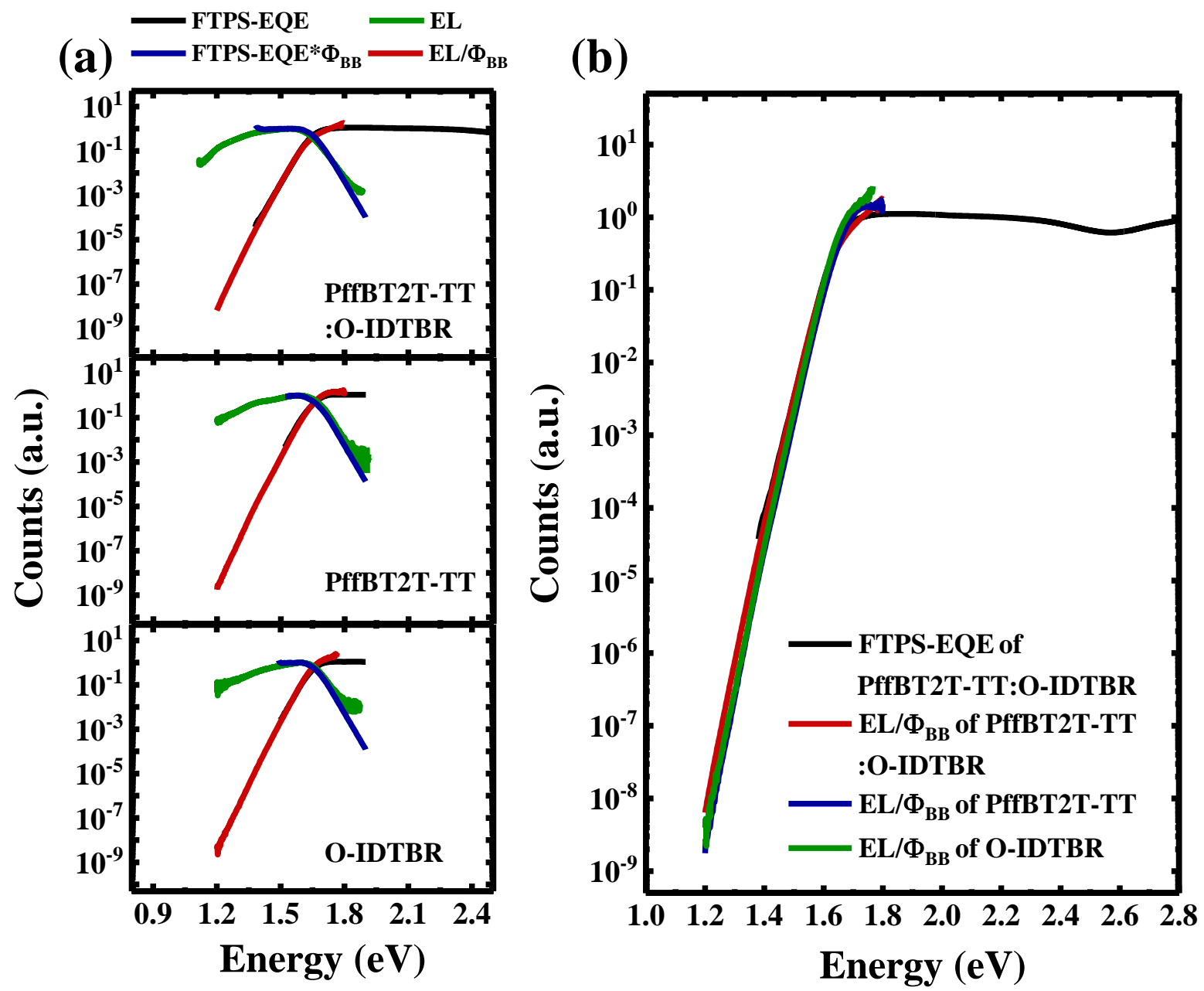

Figure 2. a) Normalized spectra of FTPS-EQE, EL, FTPS-EQE* $\Phi_{\mathrm{BB}}$ and EL/ $\Phi_{\mathrm{BB}}$. Here, $\Phi_{\mathrm{BB}}$ is the black-body spectum at $300 \mathrm{~K}$. b) Comparison of the normalized FTPS-EQE spectrum of blend devices and EL/ $\Phi_{\mathrm{BB}}$ of both neat and blend devices.

To reveal the origins of the small $V_{\text {loss }}$, FTPS-EQE and EL measurements were performed on both neat and blend based devices (Figure 2). It is well accepted that the $V_{\text {loss }}$ for any type of solar cells can be attributed to three factors: ${ }^{[17,20]}$

$$
q V_{\text {loss }}=E_{\text {gap, } \mathrm{D} / \mathrm{A}}-q V^{\mathrm{SQ}}+q V_{\text {loss,rad-below gap }}+q V_{\text {loss,non-rad }}
$$

where $q$ is the elementary charge, $V^{\mathrm{SQ}}$ is the maximum voltage determined by the Shockley-

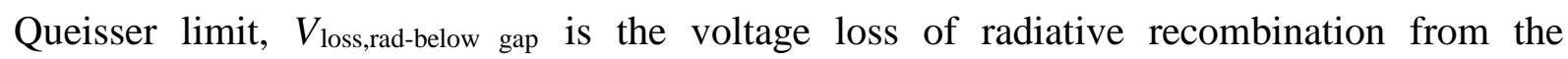
absorption below the bandgap, and $V_{\text {loss,non-rad }}$ is the voltage loss of non-radiative 
recombination. The first part $\left(E_{\text {gap }}-q V^{S \mathrm{Q}}\right)$ originates from the radiative recombination of the absorption above the bandgap. This loss is unavoidable within the Shockley-Queisser framework, limiting the maximum achievable $V_{\text {ос }}$ to $\sim 0.30 \mathrm{~V}$ below the optical bandgap $(0.28$ $\mathrm{V}$ in this work). ${ }^{[30]}$

The second voltage loss ( $\left.V_{\text {loss,rad-below gap }}\right)$ is due to the non-step-functionalized EQE of the real devices, and it is mainly contributed from the additional radiative recombination below the bandgap. ${ }^{[32]}$ Compared to inorganic and perovskite solar cells, this type of voltage loss is usually quite high for OSCs $\left(0.20-0.70 \mathrm{~V}\right.$ for state-of-the-art OSCs), ${ }^{[20]}$ as the excitons in OSCs have to undergo CT states before dissociating into free charges. Therefore, to reduce the $V_{\text {loss,rad-below gap }}$ in OSCs, it is essential to minimize the energy offset of $E_{\text {gap, D }}-E_{\text {CT }}$ or $E_{\text {gap , A }}-E_{\text {CT. }}$ To investigate both $E_{\text {gap, D }}-E_{\text {CT }}$ and $E_{\text {gap, A }}-E_{\text {CT }}$ offsets, FTPS-EQE and EL measurements were performed on the neat and blend devices as presented in Figure 2a. For clarity, the FTPS-EQE spectra of three types of devices are presented in Figure S2, and it is clearly observed that the FTPS-EQE spectrum of the blend overlaps with those of neat PffBT2T-TT and O-IDTBR. We also examine the reciprocity relation between photovoltaic quantum efficiency and electroluminescent emission of these solar cells. ${ }^{[33]}$ As shown in Figure 2a, the FTPS-EQE and EL spectra obtained from the measurements can be well reproduced by $\mathrm{EL} / \Phi_{\mathrm{BB}}$ and $\mathrm{FTPS}-\mathrm{EQE}^{*} \Phi_{\mathrm{BB}}$ in all three cases, validating the reliability of these measurements. As compared in Figure 2b, the absorption onset of the blend based devices almost overlaps with those of the neat PffBT2T-TT and O-IDTBR based devices, and

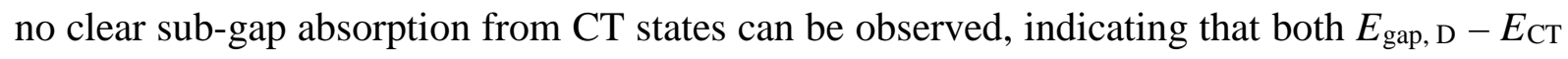

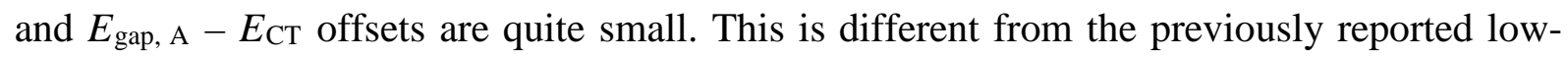
$V_{\text {loss }}$ systems like P3TEA:SF-PDI 2 . In the case of P3TEA:SF-PDI 2 , the FTPS-EQE curve of the blend only overlaps with the neat P3TEA based device yet there is still a large energy offset between the neat SF-PDI 2 and blend devices, which indicate a small $E_{\text {gap, D }}-E_{\mathrm{CT}}$ but a 


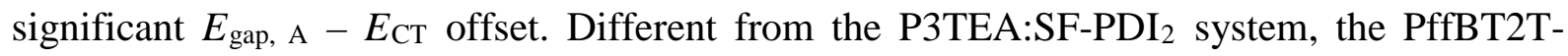
TT:O-IDTBR system can achieve both small $E_{\text {gap, D }}-E_{\text {CT }}$ and $E_{\text {gap, A }}-E_{\text {CT }}$ offsets. Taking

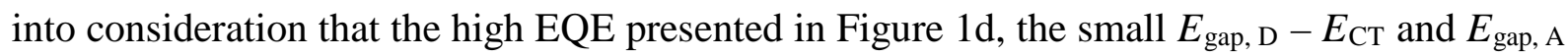
$-E_{\mathrm{CT}}$ offsets suggest that the efficient charge transfer process can occur despite small driving forces for both hole and electron transfers. The $V_{\text {loss,rad-below gap of the PffBT2T-TT:O-IDTBR- }}$ based devices is estimated to be $\sim 40 \mathrm{mV}$, much smaller than that of state-of-the-art OSCs, and comparable to that of inorganic or perovskite solar cells.

The third term of $V_{\text {loss,non-rad }}\left(=-k T \ln \left(\mathrm{EQE}_{\mathrm{EL}}\right) / q\right)$ is due to the non-radiative recombination of OSCs, where $k$ is the Boltzmann constant, $T$ is temperature and $\mathrm{EQE}_{\mathrm{EL}}$ is radiative quantum efficiency of solar cells when charge carriers are injected into the device in the dark. Therefore, the $V_{\text {loss,non-rad }}$ can be minimized by maximizing EQEEL For $c$-Si and perovskite solar cells, the $V_{\text {loss,non-rad }}$ was characterized to be 0.18 and $0.10 \mathrm{~V}$ due to their high electroluminescence efficiency, respectively. ${ }^{[20,34]}$ However, OSCs typically suffer from low EQEeL $_{\text {e }}$ thus higher $V_{\text {loss,non-rad. For example, the EQE }}$ EL for P3HT or PTB7 based OSCs is on the order of $10^{-6}$ to $10^{-8}$, corresponding to a high $V_{\text {loss,non-rad }}$ of $\sim 0.4 \mathrm{~V} \cdot{ }^{[20]}$ In contrast, the $\mathrm{EQE}_{\mathrm{EL}}$ for PffBT2T-TT-based device was measured to be as high as $\sim 1 \times 10^{-4}$, which, to our best knowledge, is one of the highest $\mathrm{EQE}_{\mathrm{EL}}$ for OSCs reported to date. The high $\mathrm{EQE}_{\mathrm{EL}}$ significantly decreases the $V_{\text {loss,non-rad }}$ to $0.24 \mathrm{~V}$, and such a small $V_{\text {loss,non-rad }}$ is approaching that of $c$-Si solar cells. The origin of reduced non-radiative recombination loss in organic solar cells with aligned energetic levels is still an open question which deserves further investigatioins. When the energetic levels between the donor and acceptor materials are aligned, the emission might be mainly from the singlet excitons which have higher quantum efficiency than that of CT states. We expect that detailed quantum chemistry calculations will shed more light on this issue, and this is beyond the scope of this article. 
It is thus clear that the high performance of this blend is enabled by efficient charge generation without the requirement of a large LUMO or HOMO energy offset (both electron and hole transfers), and the exceptionally low non-radiative recombination loss. The mechanism of charge generation in donor/acceptor organic heterojunctions is a much debated topic, with the driving energy provided by the energy offset in the molecular orbitals generally expected to play a significant role. For polymer/fullerene blends, an empirically determined minimum offset energy of $\sim 250 \mathrm{meV}$ is required to allow electrons to inject into a set of delocalized states in the fullerene crystallites, forming free carriers at ultrafast timescale. ${ }^{[35]}$ By using transient absorption (TA) spectroscopy, we can clearly observe that the charge generation in PffBT2T-TT:O-IDTBR occurs at a timescale of $\sim 10$ ps which is consistent with previous studies ${ }^{[17,36]}$ (see Figure S6 and supporting discussions in SI). However, due to overlapping absorptions between PffBT2T-TT and O-IDTBR, the analysis of the TA signals is complicated, and the precise timescale and mechanism are beyond the scope of this study. 
(a)
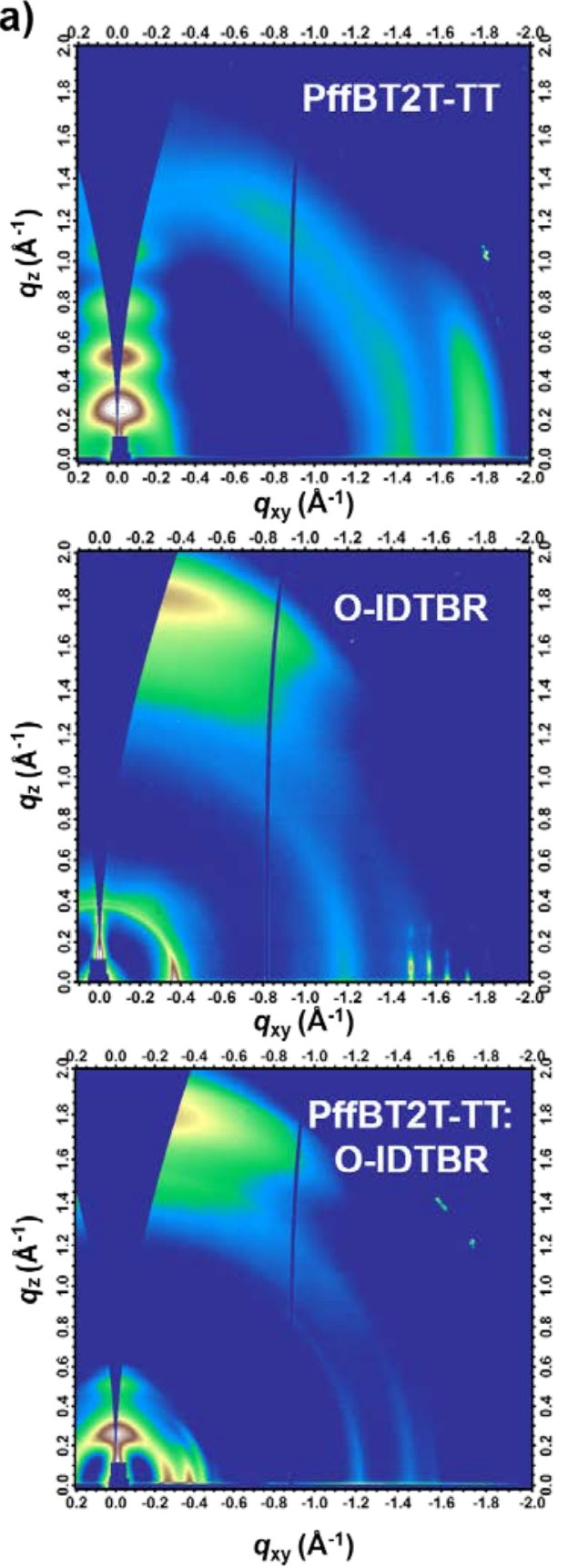

(b)

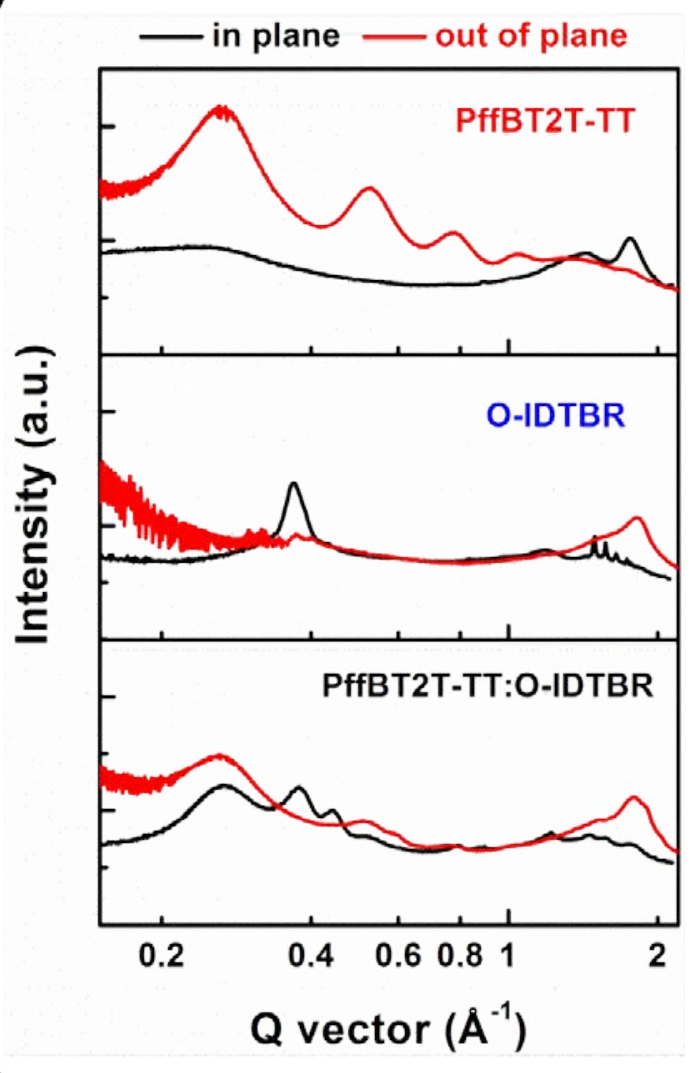

(c)

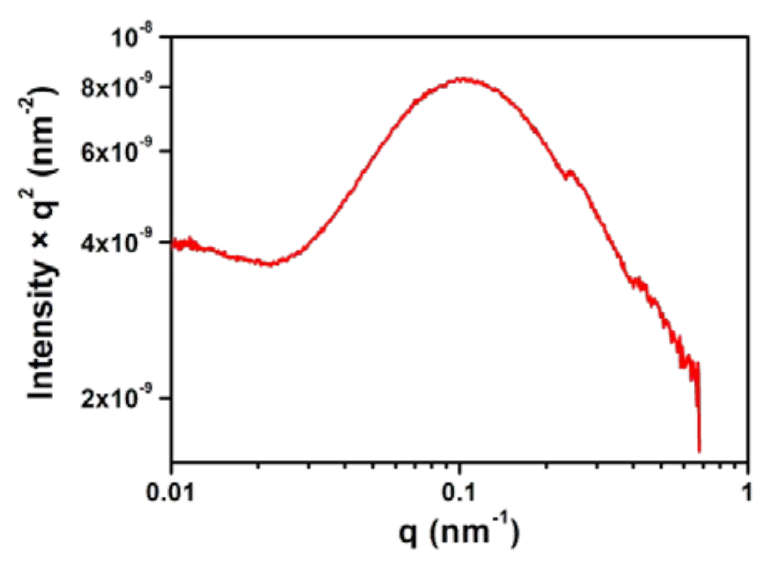

Figure 3. a) The 2D GIWAXS patterns and b) 1D profiles for PffBT2T-TT, O-IDTBR and PffBT2T-TT:O-IDTBR films. c) R-SoXS profile for the blend film.

In addition to achieving effective charge separation at the D/A interface despite the small driving forces, it is also important to achieve well-separated donor/acceptor domains with optimal transport networks to ensure that the separated holes and electrons can reach the 
anode and cathode, respectively. To investigate the morphological features of the neat and blend films based on PffBT2T-TT and O-IDTBR, both grazing incidence wide-angle X-ray scattering (GIWAXS) and resonant soft X-ray scattering (R-SoXS) were carried out (Figure 3). ${ }^{[37-44]}$ The neat PffBT2T-TT polymer exhibits high crystallinity, as high-order lamellar stacking peaks up to (400) can be clearly observed in the out-of-plane direction. The blend shows a preferential face-on orientation that is beneficial to charge transport in the vertical direction across the electrodes. ${ }^{[38,45]}$ Moreover, the stacking peaks from both PffBT2T-TT and O-IDTBR can be clearly observed in the blend film, which implies that both PffBT2T-TT and O-IDTBR can maintain their crystallinity in the blend. ${ }^{[18,26]}$ Subsequently, the phase separation of the blend was probed via resonant soft X-ray scattering (R-SoXS) and the detailed profile is presented in Figure 3c. A photon energy of $284.8 \mathrm{eV}$ was selected to probe the blends due to the highest PffBT2T-TT:O-IDTBR contrast at this energy. The domain spacing is calculated to be $60 \mathrm{~nm}$, indicating a relatively small domain size of $30 \mathrm{~nm}$ assuming a two-phase morphology with equal domain sizes, and this domain feature is consistent with the characterization results from atomic force microscopy (AFM, Figure S3) and transmission electron microscopy (TEM, Figure S4) measurements. The small domain size has been shown to be crucial for charge transporting and minimized recombination. ${ }^{[4,46]}$ Overall, a combination of morphology characterization results suggests that PffBT2T-TT:OIDTBR can achieve a favorable morphology for charge generation, including high crystallinity, face-on orientation and small domain size, thus good charge extraction and a high FF achieved in the corresponding OSCs.

In conclusion, we report an efficient non-fullerene OSC with both small driving forces for hole and electron transfers, yet still exhibits efficient charge separation. The FTPS-EQE spectrum of the blend is nearly identical to those of the neat based devices, indicating that both $E_{\text {gap, D }}-E_{\mathrm{CT}}$ and $E_{\text {gap, }}$ A $-E_{\mathrm{CT}}$ offsets are negligible. Besides, the EQE $E_{\mathrm{EL}}$ for the blend 
device is as high as $1 \times 10^{-4}$, which led to a minimized $V_{\text {loss,non-rad }}$ of $0.24 \mathrm{~V}$. Despite both small driving forces for hole and electron transfers, the PffBT2T-TT:O-IDTBR-based device can still achieve a high EQE of $67 \%$. In combination with the high crystallinity property of PffBT2T-TT and a favorable morphology, a high PCE of $10.4 \%$ was achieved with a high $V_{\text {oc }}$ of $1.08 \mathrm{~V}$ and a small $V_{\text {loss }}$ of $0.55 \mathrm{~V}$, one of the smallest $V_{\text {loss }}$ in high-performance OSCs with PCEs over $10 \%$. Fundamentally, our work is different from the previously reported low$V_{\text {loss }}$ systems like P3TEA:SF-PDI 2 , and we present the first example of efficient charge

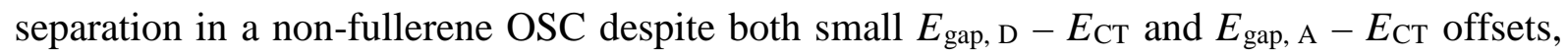
which will have fundamental significance and implications on the understanding of charge transfer processes in OSCs.

\section{Supporting Information}

Supporting Information is available from the Wiley Online Library or from the author.

\section{Acknowledgements}

The work described in this paper was partially supported by the National Basic Research Program of China (973 Program project numbers 2013CB834701 and 2014CB643501), the Shenzhen Technology and Innovation Commission (project number JCYJ20170413173814007 and JCYJ20170818113905024), the Hong Kong Research Grants Council (project numbers T23-407/13 N, N_HKUST623/13, 16305915, 16322416, 606012, 16306117 and 16303917), HK JEBN Limited, HKUST president's office (Project FP201) and the National Science Foundation of China (\#21374090), the Swedish Energy Agency Energimyndigheten (2016-010174), the Swedish Government Strategic Research Area in Materials Science on Functional Materials at Linköping University (Faculty Grant No. SFOMat-LiU \#2009-00971).We especially thank Hong Kong Innovation and Technology Commission for the support through projects ITC-CNERC14SC01 and ITS/083/15.

Received: ((will be filled in by the editorial staff))

Revised: ((will be filled in by the editorial staff)) Published online: ((will be filled in by the editorial staff))

\section{References}

[1] G. Yu, J. Gao, J. C. Hummelen, F. Wudl, A. J. Heeger, Science 1995, 270, 1789.

[2] Z. He, C. Zhong, S. Su, M. Xu, H. Wu, Y. Cao, Nat. Photonics 2012, 6, 591. 
[3] X. Guo, N. Zhou, S. J. Lou, J. Smith, D. B. Tice, J. W. Hennek, R. P. Ortiz, J. T. L. Navarrete, S. Li, J. Strzalka, L. X. Chen, R. P. H. Chang, A. Facchetti, T. J. Marks, Nat. Photonics 2013, 7, 825.

[4] J. Zhao, Y. Li, G. Yang, K. Jiang, H. Lin, H. Ade, W. Ma, H. Yan, Nat. Energy 2016, $1,15027$.

[5] Y. Lin, X. Zhan, Mater. Horiz. 2014, 1, 470.

[6] S. Li, W. Liu, C.-Z. Li, M. Shi, H. Chen, Small 2017, 13, 1701120.

[7] N. Liang, W. Jiang, J. Hou, Z. Wang, Materials Chemistry Frontiers 2017, 1, 1291.

[8] G. Sauve, R. Fernando, J. Phys. Chem. Lett. 2015, 6, 3770.

[9] S. Dai, F. Zhao, Q. Zhang, T. K. Lau, T. Li, K. Liu, Q. Ling, C. Wang, X. Lu, W. You, X. Zhan, J. Am. Chem. Soc. 2017, 139, 1336.

[10] F. Zhao, S. Dai, Y. Wu, Q. Zhang, J. Wang, L. Jiang, Q. Ling, Z. Wei, W. Ma, W. You, C. Wang, X. Zhan, Adv. Mater. 2017, 29, 1700144.

[11] S. Li, L. Ye, W. Zhao, S. Zhang, S. Mukherjee, H. Ade, J. Hou, Adv. Mater. 2016, 28, 9423.

[12] H. Bin, L. Gao, Z.-G. Zhang, Y. Yang, Y. Zhang, C. Zhang, S. Chen, L. Xue, C. Yang, M. Xiao, Y. Li, Nat. Commun. 2016, 7, 13651.

[13] B. Kan, H. Feng, X. Wan, F. Liu, X. Ke, Y. Wang, Y. Wang, H. Zhang, C. Li, J. Hou, Y. Chen, J. Am. Chem. Soc. 2017, 139, 4929.

[14] S. J. Xu, Z. Zhou, W. Liu, Z. Zhang, F. Liu, H. Yan, X. Zhu, Adv. Mater. 2017, 1704510.

[15] W. Zhao, S. Li, H. Yao, S. Zhang, Y. Zhang, B. Yang, J. Hou, J. Am. Chem. Soc. 2017, 139, 7148.

[16] D. Meng, H. Fu, C. Xiao, X. Meng, T. Winands, W. Ma, W. Wei, B. Fan, L. Huo, N. L. Doltsinis, Y. Li, Y. Sun, Z. Wang, J. Am. Chem. Soc. 2016, 138, 10184.

[17] J. Liu, S. Chen, D. Qian, B. Gautam, G. Yang, J. Zhao, J. Bergqvist, F. Zhang, W. Ma, H. Ade, O. Inganäs, K. Gundogdu, F. Gao, H. Yan, Nat. Energy 2016, 1, 16089.

[18] S. Chen, Y. Liu, L. Zhang, P. C. Y. Chow, Z. Wang, G. Zhang, W. Ma, H. Yan, J. Am. Chem. Soc. 2017, 139, 6298.

[19] P. Cheng, M. Zhang, T.-K. Lau, Y. Wu, B. Jia, J. Wang, C. Yan, M. Qin, X. Lu, X. Zhan, Adv. Mater. 2017, 1605216.

[20] J. Z. Yao, T. Kirchartz, M. S. Vezie, M. A. Faist, W. Gong, Z. C. He, H. B. Wu, J. Troughton, T. Watson, D. Bryant, J. Nelson, Phys. Rev. Appl. 2015, 4, 014020.

[21] N. A. Ran, J. A. Love, C. J. Takacs, A. Sadhanala, J. K. Beavers, S. D. Collins, Y. Huang, M. Wang, R. H. Friend, G. C. Bazan, T. Q. Nguyen, Adv. Mater. 2016, 28, 1482.

[22] K. Kawashima, Y. Tamai, H. Ohkita, I. Osaka, K. Takimiya, Nat. Commun. 2015, 6, 10085.

[23] K. Vandewal, K. Tvingstedt, A. Gadisa, O. Inganas, J. V. Manca, Nat. Mater. 2009, 8, 904.

[24] Y. Lin, F. Zhao, Y. Wu, K. Chen, Y. Xia, G. Li, S. K. K. Prasad, J. Zhu, L. Huo, H. Bin, Z.-G. Zhang, X. Guo, M. Zhang, Y. Sun, F. Gao, Z. Wei, W. Ma, C. Wang, J. Hodgkiss, Z. Bo, O. Inganäs, Y. Li, X. Zhan, Adv. Mater. 2017, 29, 1604155.

[25] H. Lin, S. Chen, Z. Li, J. Y. Lai, G. Yang, T. McAfee, K. Jiang, Y. Li, Y. Liu, H. Hu, J. Zhao, W. Ma, H. Ade, H. Yan, Adv. Mater. 2015, 27, 7299.

[26] S. Chen, H. Yao, Z. Li, O. M. Awartani, Y. Liu, Z. Wang, G. Yang, J. Zhang, H. Ade, H. Yan, Adv. Energy Mater. 2017, 7, 1602304.

[27] Q. Fan, Z. Xu, X. Guo, X. Meng, W. Li, W. Su, X. Ou, W. Ma, M. Zhang, Y. Li, Nano Energy 2017, 40, 20.

[28] Y. Li, D. Qian, L. Zhong, J.-D. Lin, Z.-Q. Jiang, Z.-G. Zhang, Z. Zhang, Y. Li, L.-S. Liao, F. Zhang, Nano Energy 2016, 27, 430. 
[29] S. Holliday, R. S. Ashraf, A. Wadsworth, D. Baran, S. A. Yousaf, C. B. Nielsen, C. H. Tan, S. D. Dimitrov, Z. Shang, N. Gasparini, M. Alamoudi, F. Laquai, C. J. Brabec, A. Salleo, J. R. Durrant, I. McCulloch, Nat. Commun. 2016, 7, 11585.

[30] S. M. Menke, N. A. Ran, G. C. Bazan, R. H. Friend, Joule 2018, 2, 25.

[31] J. Hou, O. Inganäs, R. H. Friend, F. Gao, Nat. Mater. 2018, 17, 119.

[32] U. Rau, B. Blank, T. C. M. Muller, T. Kirchartz, Phys. Rev. Appl. 2017, 7, 044016.

[33] U. Rau, Phys. Rev. B 2007, 76, 085303.

[34] M. Saliba, T. Matsui, K. Domanski, J. Y. Seo, A. Ummadisingu, S. M. Zakeeruddin, J. P. Correa-Baena, W. R. Tress, A. Abate, A. Hagfeldt, M. Gratzel, Science 2016, 354, 206.

[35] S. Gelinas, A. Rao, A. Kumar, S. L. Smith, A. W. Chin, J. Clark, T. S. van der Poll, G. C. Bazan, R. H. Friend, Science 2014, 343, 512.

[36] S. M. Menke, A. Cheminal, P. Conaghan, N. A. Ran, N. C. Greehnam, G. C. Bazan, T.-Q. Nguyen, A. Rao, R. H. Friend, Nat. Commun. 2018, 9, 277.

[37] B. A. Collins, J. E. Cochran, H. Yan, E. Gann, C. Hub, R. Fink, C. Wang, T.

Schuettfort, C. R. McNeill, M. L. Chabinyc, H. Ade, Nat. Mater. 2012, 11, 536.

[38] J. R. Tumbleston, B. A. Collins, L. Q. Yang, A. C. Stuart, E. Gann, W. Ma, W. You, H. Ade, Nat. Photonics 2014, 8, 385.

[39] W. Ma, J. R. Tumbleston, M. Wang, E. Gann, F. Huang, H. Ade, Adv. Energy Mater. 2013, 3, 864.

[40] S. Mukherjee, C. M. Proctor, G. C. Bazan, T.-Q. Nguyen, H. Ade, Adv. Energy Mater. 2015, 5, 1500877.

[41] S. Mukherjee, C. M. Proctor, J. R. Tumbleston, G. C. Bazan, T. Q. Nguyen, H. Ade, Adv. Mater. 2015, 27, 1105.

[42] B. A. Collins, Z. Li, J. R. Tumbleston, E. Gann, C. R. McNeill, H. Ade, Adv. Energy Mater. 2013, 3, 65.

[43] P. Muller-Buschbaum, Adv. Mater. 2014, 26, 7692.

[44] E. Gann, A. T. Young, B. A. Collins, H. Yan, J. Nasiatka, H. A. Padmore, H. Ade, A. Hexemer, C. Wang, Rev. Sci. Instrum. 2012, 83, 045110.

[45] V. Vohra, K. Kawashima, T. Kakara, T. Koganezawa, I. Osaka, K. Takimiya, H. Murata, Nat. Photonics 2015, 9, 403.

[46] Y. Zang, C. Z. Li, C. C. Chueh, S. T. Williams, W. Jiang, Z. H. Wang, J. S. Yu, A. K. Jen, Adv. Mater. 2014, 26, 5708. 
An efficient non-fullerene OSC is realized by combining a donor polymer named PffBT2TTT and a small-molecular acceptor (O-IDTBR) with identical bandgaps and close energy levels. Despite the small energy offsets for both hole and electron transfers, this system can still achieve efficient charge separation and a high efficiency of $10.4 \%$.

Keyword: organic solar cells, small-molecular acceptors, voltage loss, charge transfer

Shangshang Chen, Yuming Wang, Lin Zhang, Jingbo Zhao, Yuzhong Chen, Danlei Zhu, Huatong Yao, Guangye Zhang, Wei Ma, Richard. H. Friend, Philip C. Y. Chow*, Feng Gao,* and He Yan*

\section{Efficient Non-Fullerene Organic Solar Cells with Small Driving Forces for Both Hole and Electron Transfers}

ToC figure ((Please choose one size: $55 \mathrm{~mm}$ broad $\times 50 \mathrm{~mm}$ high or $110 \mathrm{~mm}$ broad $\times 20 \mathrm{~mm}$ high. Please do not use any other dimensions))

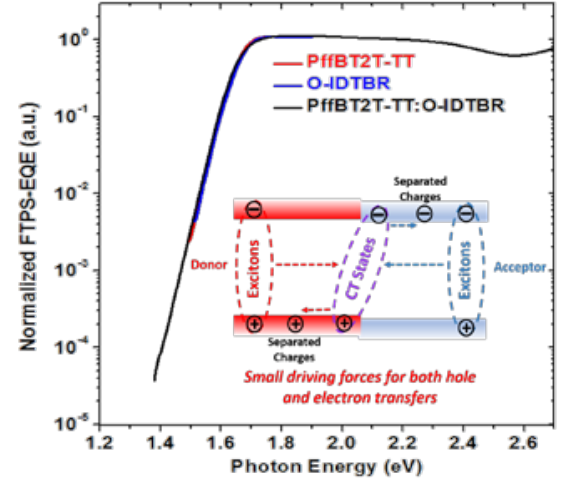

